\title{
Efficient hearing aid algorithm using DCT with uniformly re-sampled and recursively modified audiogram values
}

\author{
K. Ayyappa Swamy ${ }^{1}$, Zachariah C. Alex ${ }^{2}$ \\ ${ }^{1,2}$ School of Electronics Engineering, Vellore institute of Technology, Vellore, Tamilnadu, India \\ ${ }^{1}$ Bio signal Research Lab, SreeVidyanikethan Engineering College, Tirupati, Andhra Pradesh, India
}

\begin{tabular}{l}
\hline Article Info \\
\hline Article history: \\
Received Feb 16, 2021 \\
Revised Jun 7, 2021 \\
Accepted Jun 17, 2021 \\
\hline
\end{tabular}

Keywords:

Audiogram re-sampling

Discrete cosine transform

Gain adjustment

Hearing aid

Sensorineural hearing loss

\begin{abstract}
People with the hearing problems have different listening preferences and characteristics in hearing loss. So, hearing aids need algorithms that provide amplification based on frequency, so that the hearing-impaired persons can use hearing aids comfortably for a long duration. In this paper, a new algorithm is proposed for hearing aids in order to compensate for sensorineural and conductive hearing loss using discrete cosine transform (DCT). DCT coefficients of the input audio signal are multiplied with uniformly resampled and recursively modified audiogram values to compensate for hearing loss. This algorithm comprised of 4 stages namely precomputation to calculate gain values from audiogram, DCT, gain adjustment, and inverse DCT. In the above stated stages except precomputation, each stage requires only one matrix multiplication, which makes the proposed algorithm computational efficient. Performance of the proposed algorithm is compared with uniform filter banks, non-uniform filter banks, variable filter bank and reconfigurable filter banks. The algorithm is tested using audiograms with four different hearing loss cases. It is proved that the proposed algorithm provides less complexity, minimized delay and better matching with all types of audiograms, further, it also avoids degradation of audio signal due to sampling rate conversions in variable and reconfigurable filter banks.
\end{abstract}

This is an open access article under the CC BY-SA license.

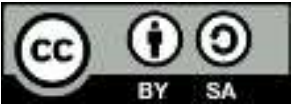

Corresponding Author:

Zachariah C. Alex

School of Electronics Engineering

Vellore institute of Technology, Vellore

Tamilnadu, 632014, India

Email: zachariahcalex@vit.ac.in

\section{INTRODUCTION}

Elderly people may not hear properly due to damaged nerve fibers and sensory cells of the inner ear [1]. Hearing aid may be used to compensate for this disability. The hearing aid is an electro acoustic device that amplifies sound signals to compensate for hearing loss. However, characteristics of hearing loss vary from person to person based on the hearing thresholds. It is considered that, normal hearing is between -10 to $20 \mathrm{~dB}$, the mild hearing loss occurs between 20 to $40 \mathrm{~dB}$, moderate is between 40 to $55 \mathrm{~dB}$, moderately severe is between 55 to $70 \mathrm{~dB}$ and severe is 70 to $90 \mathrm{~dB}$ or profound greater than $90 \mathrm{~dB}$ [2], [3]. With a sensorineural hearing loss, one might lose only a certain band of frequency [4]. Thus, Normal hearing aid uniformly amplifies all frequencies in audio signals, but it needs to amplify only the sounds that can't be hear by hearing-impaired, if not the louder sounds become unbearable [5]. Therefore, in hearing aids, a particular band of frequencies of audio signals are subjected for suitable gain adjustment based on an audiogram to make the person understand the speech. 
In the present scenario, the research is carried on in the implementation of a signal processing algorithm to compensate for different types of hearing losses. Current studies focus on filter bank structures with less computational complexity in order to reduce the hardware complexity and also to increase the speed of operation. Foremost, and mostly used filter banks are uniform filter bank [6]-[8] and nonuniform filter bank [9]-[11]. Over the past decade, the researchers implemented distinguished efficient variable filter bank structures [12], [13] and reconfigurable filter banks [14]-[20] to get better matching with an audiogram and to reduce the computational complexity of filter banks.

Considerably better match between the frequency response of the hearing aid and audiogram is achievable if a greater number of bands are assigned for a uniform and non-uniform filter bank. But a few drawbacks can be observed such as delay, power consumption, and the size of the hearing aid increase with the increase in the number of bands. In case of uniform and non-uniform filter banks one should compromise on either size and delay or matching error. To achieve better matching error with smaller delay and size filter bank structure should vary with type of the audiogram. Whereas, in uniform and non-unifrom filter banks the filter bank structure is fixed for all types of audiograms. To overcome these drawbacks, reconfigurable filter banks are introduced. In a reconfigurable filter bank the number of subbands in each band varies based on some parameters which gives different structures for different types of audiograms. Even in reconfigurable filter banks a shortcoming is observed, that it uses interpolation and decimation of filter coefficients and/or input signal to convert the sampling rate, which results in signal degradation and also, aliasing effect may occur due to sampling rate conversion. To overcome this hindering, the present research proposes a new technique in which the gains are adjusted in the frequency domain using DCT.

In this technique, DCT coefficients are multiplied with uniformly re-sampled and recursively modified audiogram values to adjust gain in frequency domain, after gain adjustment the frequency domain signal is converted back to time domain using inverse DCT. Audiogram values are re-sampled at uniform intervals of frequency and they are modified to get minimum matching error. This precomputation is performed for each audiogram before loading gain values into the hearing aid. The proposed DCT based algorithm is better when compared to fixed filter banks and reconfigurable filter banks. This is better in terms of complexity as it has only 3 matrix multiplications to perform gain adjustment in the frequency domain. It gives better matching error as it uses recursive modifications of audiogram values based on matching error. This DCT based technique need preprocessing to get gain values from audiogram which is not required in filter bank structures that is the only disadvantage of the proposed technique. Audiogram preprocessing is performed before loading the gain values into the processor. So, it won't affect the speed of the hearing aid system.

The proposed algorithm provides a simple solution to compensate for the hearing loss without any filter banks and sampling rate conversions. Totally 3 stages are needed for the whole process i) finding DCT for the input audio signal, ii) gain adjustment and iii) inverse DCT. The proposed algorithm is tested using audiograms with four different hearing loss cases such as mild hearing loss at high frequencies, mild to moderate hearing loss at low frequencies, moderate hearing loss at middle frequencies and mild conductive hearing loss. It is noted that the proposed algorithm provides less complexity, less delay and better matching with audiogram with all types of audiograms. It also avoids degradation of audio signal due to sampling rate conversions that are used in variable and reconfigurable filter banks.

The paper is organized as follows: Section 2 deals with the implementation of the proposed DCT based algorithm. Section 3 discusses the precomputation to find the gain values from audiogram. In section 4, design examples and performance evaluation proposed algorithm are tested with audiograms with four different hearing loss cases. Section 5 brings in experimental results and analysis. Finally, the conclusion is drawn in section 6.

\section{PROPOSED DCT BASED ALGORITHM}

In this proposed technique, the audio signal is transformed to the frequency domain using DCT to amplify the audio signal as per the above requirement. The commonly used transform domain approaches are based on DCT [21]. DCT is used to convert the data into a sum of cosine wave trays of different frequencies. As the DCT coefficients are arranged in ascending order with respect to their corresponding frequencies, it is very easy to adjust the gains. Uniformly re-sampled and recursively modified audiogram values are multiplied with the DCT coefficients of the audio signal to perform the gain adjustment. Audiogram re-sampling is discussed in the precomputation section of this paper. Figure 1 represents the block diagram of the proposed algorithm. As per the block diagram, input audio signal sensed by microphone is given to analog to digital converter (ADC) which converts the analog audio signal into a digital audio signal. Then, that digital signal is transformed into the frequency domain using DCT transform. Output of the DCT block is DCT coefficients. Further, in the gain adjustment block DCT coefficients are multiplied with uniformly 
re-sampled and recursively modified audiogram values. Then, inverse DCT transforms the amplified DCT coefficients into time domain. Finally, digital to analog converter (DAC) is to convert the digital auditory compensated signal to analog and given to the audio speaker.

Figure 2 (a) explains the working procedure of the proposed algorithm. Consider the sampling frequency of the input audio signal Fs $=16 \mathrm{kHz}$. In order to apply 80-point DCT on input signal 80 input samples need to be stored in the buffer. Then, apply 80-point DCT to the input samples stored in buffer to convert the time domain signal into frequency-domain. Later, in Figure 2 (b) the DCT coefficients are multiplied with uniformly re-sampled and recursively modified audiogram values to adjust gain values. Thus, to convert the amplified signals back to time-domain, apply 80-point inverse DCT. Now the output is time domain audio signal after gain adjustment.

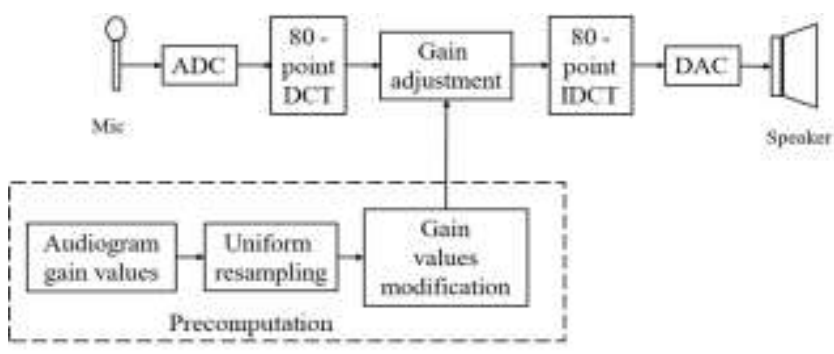

Figure 1. Block diagram for proposed algorithm

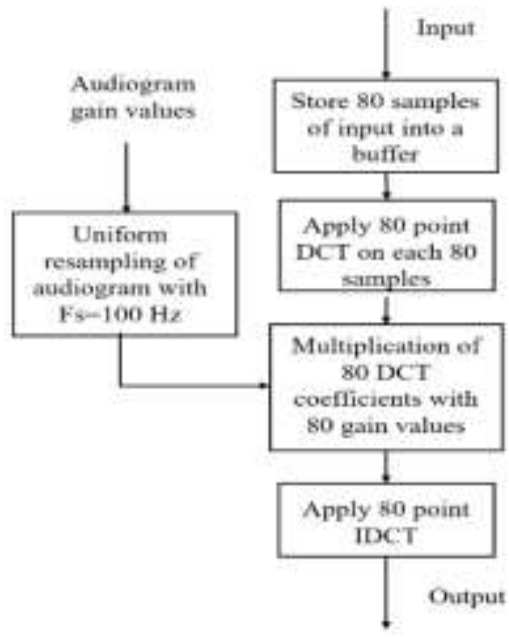

(a)

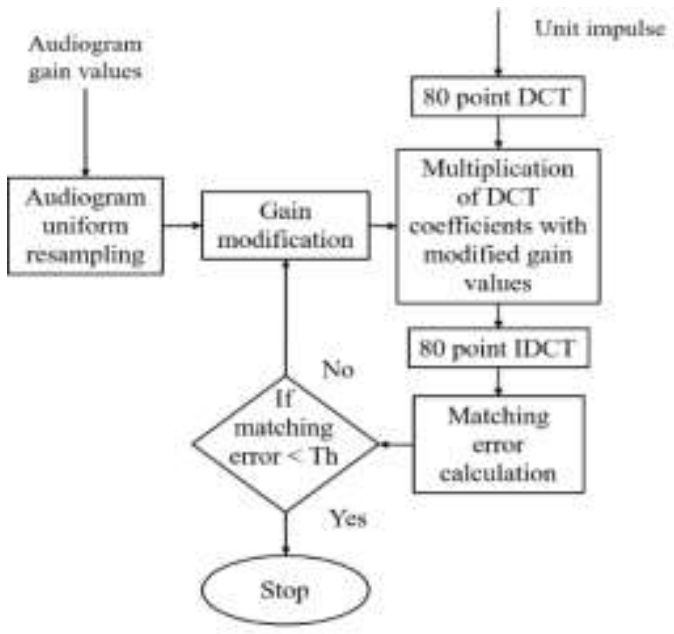

(b)

Figure 2. These figures are; (a) working procedure of the proposed algorithm, and (b) modifying audiogram values recursively

\section{PRECOMPUTATION}

This section discusses how audiogram values are re-sampled and modified recursively in order to find the gain values that are needed to be adjusted in frequency domain.

\subsection{Audiogram re-sampling}

Graph in Figure 3 (a) represents the audiogram values for the hearing loss case with mild hearing loss at mid frequency. Graph in Figure 3 (b) is the uniformly re-samples audiogram values at $100 \mathrm{~Hz}$ that mean sampling interval in frequency domain is $100 \mathrm{~Hz}$. From the audiogram in Figure 4 (a), it is observed that the audiogram is recorded at non-uniform frequencies. In order to get uniformly sampled audiogram values from the above audiogram, it is needed to be re-sampled at uniform intervals of frequency. This can be implemented by a simple computer application by the audiologist before programming the digital hearing aid. Interpolation is needed for re-sampling of an audiogram and interpolated samples should be an average of previous and next sample values. As the audiogram is recorded at non-uniform frequencies it may require non-uniform interpolation; for example, if the new sampling interval in frequency domain is $100 \mathrm{~Hz}$ that 
means if the audiogram needs to be re-sampled at every $100 \mathrm{~Hz}$ then between $500 \mathrm{~Hz}$ and $1000 \mathrm{~Hz} 4$ samples need to be interpolated, whereas between $4000 \mathrm{~Hz}$ and $8000 \mathrm{~Hz} 39$ samples are to be interpolated. This non-uniform re-sampling of the audiogram is explained in the equation.

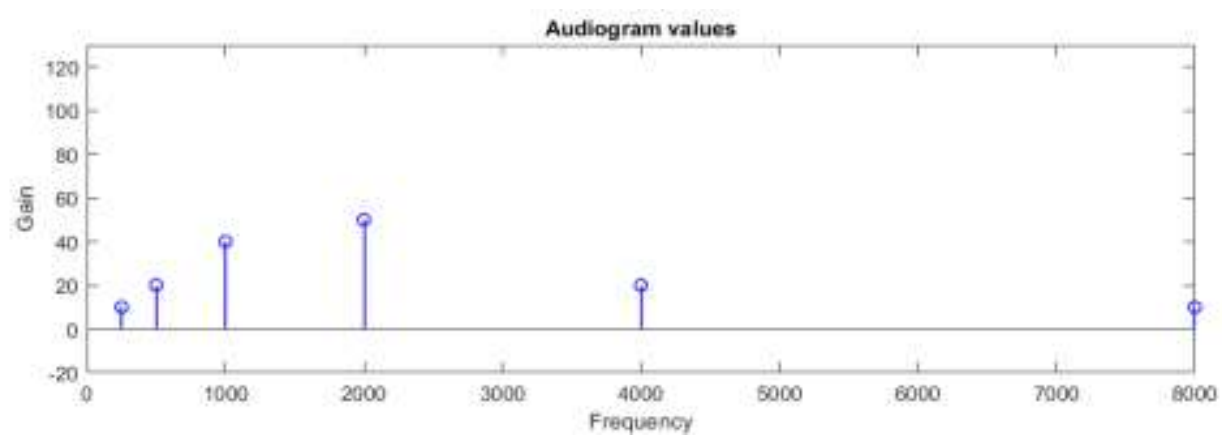

(a)

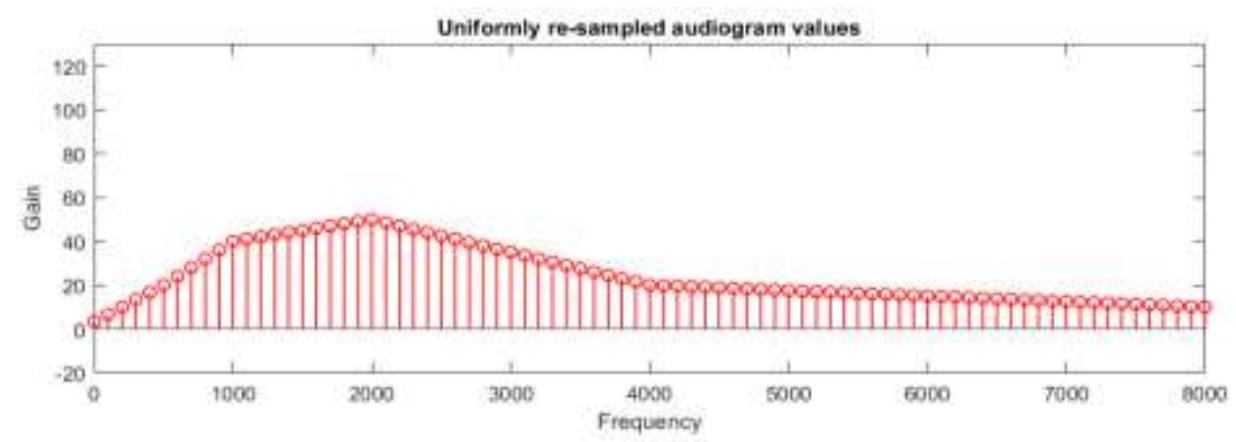

(b)

Figure 3. These figures are; (a) audiogram values, and (b) uniformly re-sampled audiogram values.

In (2) $F(k)$ contains the frequencies at which the audiogram is recorded. In (1), $G(k)$ contains the gain values in the audiogram corresponding to the frequencies defined in the $\mathrm{F}(\mathrm{k})$. $\mathrm{N}$ is the index of the current interpolating sample among the $\mathrm{N}$ number of samples. $\mathrm{N}$ is the number of samples to be interpolated between $(\mathrm{k}-1)^{\text {th }}$ and $\mathrm{k}^{\text {th }}$ samples of the audiogram. $\mathrm{F}_{\mathrm{d}}$ is the frequency difference between two int-erpolated samples (new uniform sampling interval in frequency domain) $F_{d}=100$. Elements in $G_{n}(n, k)$ are expanded into a single vector of size 80 for $\mathrm{F}_{\mathrm{d}}=100$.

$$
G_{n(k, n)}=G(k-1)+\frac{[G(k)-G(k-1)] n}{N}
$$

where:

$$
\begin{aligned}
& N=\text { round }\left[\frac{F(k)-F(k-1)}{F_{d}}\right] \\
& F(0)=G(0)=0
\end{aligned}
$$

\subsection{Recursive modification of audiogram values}

The proposed algorithm can be implemented directly but the matching error between audiogram and frequency response of the designed system is very high in some cases like moderate sensorineural hearing loss. To minimize this matching error, modification of gain values (audiogram re-sampled values) using a recursive algorithm is proposed. In this algorithm, the weighted matching error is recursively added to the uniformly re-sampled audiogram values. This process may be repeated till the matching error is reduced to minimum level. The number of iterations and the weight values depend on the type of audiogram. By trial and error, it is observed that the weight value is in between 0.1 and 1 . Gain values are loaded into the hearing aid after re-sampling and modification. To generate modified gain values for the given audiogram this 
algorithm is implemented on PC. Changes in matching error concerning the number of iterations and weight is explained in detail in the section $\mathrm{V}$ experimental results and analysis.

The working procedure for recursively modifying audiogram gain values is shown in Figure 2 (b). In this process, gain values from audiogram are subjected to the uniformly re-sampling block to obtain uniformly re-sampled audiogram values. These gain values are recursively modified based on the matching error, if matching error is less than predefined threshold value then it undergoes one more iteration. Gain modification in this algorithm implies updating gain values with the weighted matching error

\section{DESIGN EXAMPLES AND PERFORMANCE EVALUATION}

The idea of the proposed algorithm for hearing aid is examined by using some examples. The performance of the proposed algorithm is evaluated using audiograms with four types of hearing loss cases. Based on levels of hearing thresholds, hearing losses are categorized as mild, moderate, moderately severe, severe and profound. Hearing loss cases like severe and profound may not be compensated using a hearing aid [22].

\subsection{Example 1: Audiogram for mild hearing loss at high frequencies}

The audiogram for mild hearing loss at high frequencies is shown in Figure 4 (a). The right ear hearing thresholds represented by 'O' are considered for compensation. According to the audiogram gain values are 5,5,5,5,35,5 $\mathrm{dB}$. Gain values are given to the re-sampling and recursively modification block, then obtained 80 uniformly sampled gain values at the output. Thus, these 80 gain values are provided to gain adjustment block. Gains are multiplied with the DCT coefficients of the audio signal in the gain adjustment block. After gain adjustment, apply inverse DCT to convert amplified DCT coefficients back to time domain. Figure 5 (a) shows the audiogram values and the frequency response of the hearing aid system. Figure 6 (a) represents the matching error between the re-sampled audiogram and the frequency response of the hearing aid system. Matching error is the difference between re-sampled audiogram values and the frequency response of the hearing aid system. From the above Figures 5 (a) and 6 (a) and Table 1, it is clear that the proposed algorithm performs better in terms of maximum matching error and delay, when compared with filter bank techniques. Maximum matching error is the maximum difference between audiogram and frequency response of the hearing aid system. From Table 2 the proposed DCT based algorithm gives 0.49 $\mathrm{dB}$ matching error at 20 iterations and with weight 0.7 .

Table 1. Comparison of the proposed algorithm with the filter bank structures

\begin{tabular}{|c|c|c|c|c|c|c|c|c|c|c|c|c|}
\hline \multirow[t]{2}{*}{ Filter bank } & \multirow[b]{2}{*}{$\begin{array}{c}\text { Number } \\
\text { of side } \\
\text { bands }\end{array}$} & \multirow[b]{2}{*}{$\begin{array}{c}\text { Example } 1 \\
\text { Maximum } \\
\text { Matching } \\
\text { Error (dB) }\end{array}$} & \multirow[b]{2}{*}{$\begin{array}{c}\text { Delay } \\
(\mathrm{ms})\end{array}$} & \multirow[b]{2}{*}{$\begin{array}{l}\text { Numbe } \\
\text { rof } \\
\text { side } \\
\text { bands }\end{array}$} & \multirow[b]{2}{*}{$\begin{array}{l}\text { Example } 2 \\
\text { Maximum } \\
\text { Matching } \\
\text { Error (dB) }\end{array}$} & \multirow[b]{2}{*}{$\begin{array}{c}\text { Delay } \\
(\mathrm{ms})\end{array}$} & \multicolumn{3}{|c|}{ Example 3} & \multicolumn{3}{|c|}{ Example 4} \\
\hline & & & & & & & $\begin{array}{c}\text { Number } \\
\text { of side } \\
\text { bands }\end{array}$ & $\begin{array}{c}\text { Maximu } \\
\mathrm{m} \\
\text { Matchin } \\
\mathrm{g} \text { Error } \\
\text { (dB) }\end{array}$ & $\begin{array}{l}\text { De } \\
\text { lay } \\
(\mathrm{m} \\
\mathrm{s})\end{array}$ & $\begin{array}{l}\text { Number } \\
\text { of side } \\
\text { bands }\end{array}$ & $\begin{array}{c}\text { Maximum } \\
\text { Matching } \\
\text { Error (dB) }\end{array}$ & $\begin{array}{l}\text { Delay } \\
(\mathrm{ms})\end{array}$ \\
\hline $\begin{array}{l}\text { Direct } \\
\text { design }\end{array}$ & 8 & 6.39 & 4.3 & - & - & - & - & - & - & 8 & 5.86 & 4.3 \\
\hline [10] & 10 & 9.61 & 15.7 & 8 & 3.2 & 5.7 & 8 & 9.2 & $\begin{array}{l}15 \\
.7\end{array}$ & 10 & 3.67 & 5.7 \\
\hline [11] & 16 & 2.10 & 12.8 & - & - & - & - & - & - & - & - & - \\
\hline [15] & 8 & 4.82 & 29 & - & - & - & - & - & - & 7 & 2.67 & 25 \\
\hline [23] & 7 & 5.63 & 12.1 & - & - & - & - & - & - & 7 & 1.84 & 2.1 \\
\hline [17] & 10 & 2.84 & 6.6 & 12 & 1.51 & 12 & 13 & 2.72 & 18 & 11 & 1.49 & 12 \\
\hline [19] & 6 & 2.84 & $\begin{array}{c}15.7 \\
5\end{array}$ & 12 & 1.49 & 12 & 13 & 2.72 & 18 & 7 & 1.36 & 1.09 \\
\hline $\begin{array}{l}\text { Proposed } \\
\text { algorithm }\end{array}$ & - & 1 & 7.4 & - & 0.88 & 7.4 & - & 1.87 & $\begin{array}{l}7 . \\
4\end{array}$ & - & 0.15 & 7.4 \\
\hline
\end{tabular}

\subsection{Example 2: Audiogram for mild to moderate hearing loss at low frequencies}

According to the audiogram shown in Figure 4 (b) the gain values are 45, 35, 20, 10, 5, 10 respectively. Figure 5 (b) represents the audiogram values and the frequency response of the hearing aid system. Figure 6 (b) represents the matching error. From Figures 5 (b), 6 (b), and Table 1 it is clear that the proposed algorithm performs better in terms of maximum matching error and delay, when compared with filter bank techniques. DCT based algorithm gives $0.88 \mathrm{~dB}$ matching error at 20 iterations and with weight 0.3 . 


\subsection{Example 3: Audiogram for moderate hearing loss at middle frequencies}

According to the audiogram shown in Figure 4 (c) the gain values are 10, 20, 40, 50, 20, and 10 respectively. Figure 5 (c) shows the audiogram values and the frequency response of the hearing aid system. Figure 6 (c) represents the matching error. From the above results, it is clear that the proposed DCT based algorithm gives $1.87 \mathrm{~dB}$ matching error at 20 iterations and with weight 0.4

\subsection{Example 4: Audiogram for mild conductive hearing loss}

According to the audiogram shown in Figure 4 (d) the gain values are 25, 25, 25, 35, 25, and 30 respectively. Figure 5 (d) shows the audiogram values and the frequency response of the hearing aid system. Figure 6 (d) represents the matching error. It is clear from the above stated Figures 4 (d), 5 (d), 6 (d), and Table 1 that the proposed algorithm performs better when compared with all filter bank techniques. From Table 2 the proposed DCT based algorithm gives $0.15 \mathrm{~dB}$ matching error at 20 iterations and with weight 1 . From the above examples, it is evident that the DCT based auditory compensation is simple to implement and has only 80 multipliers in each of three stages namely DCT, gain adjustment and inverse DCT. The matching error is minimum when compared with the fixed filter bank and the reconfigurable filter bank

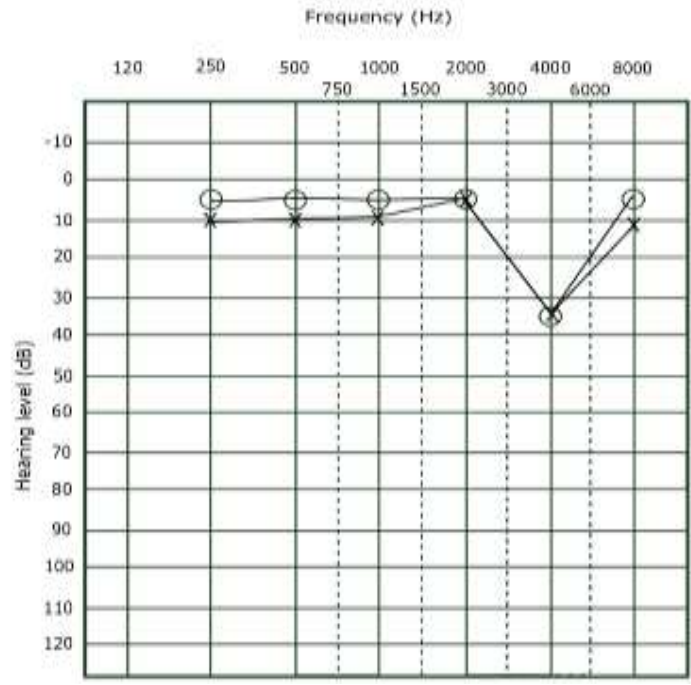

(a)

Frequency (He)

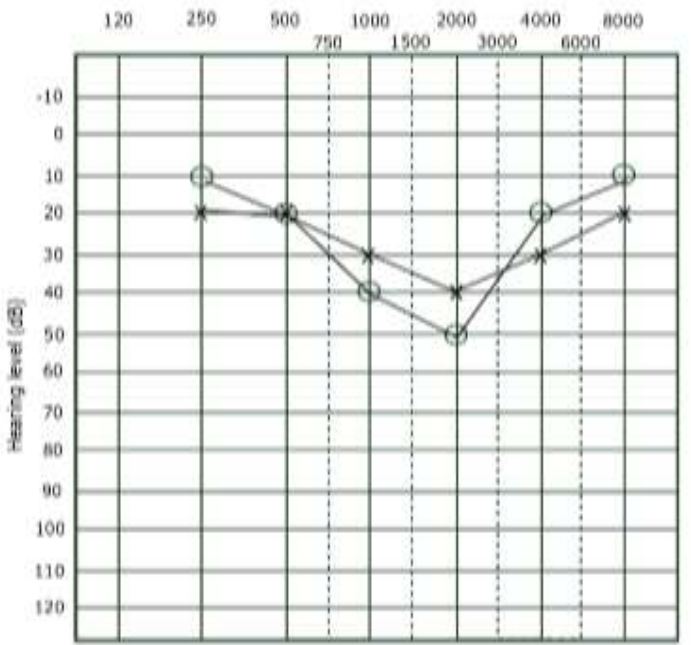

(c)

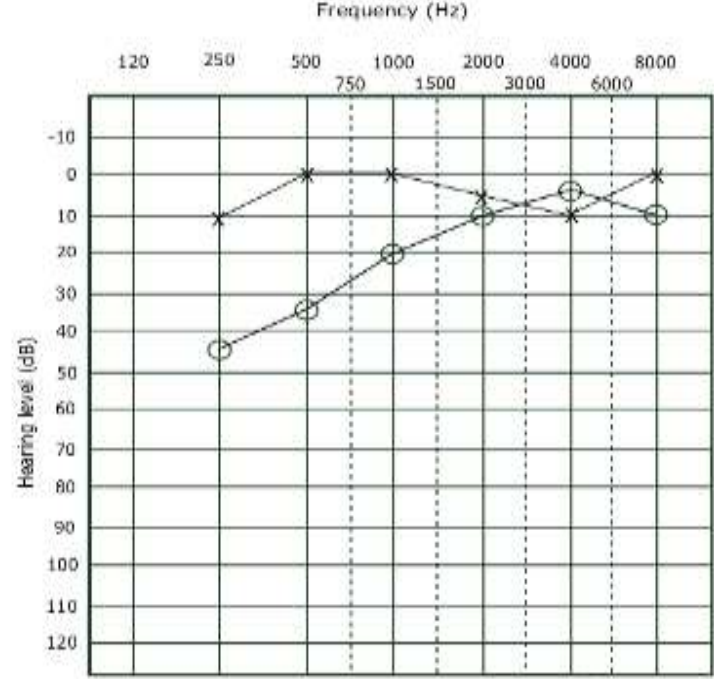

(b)

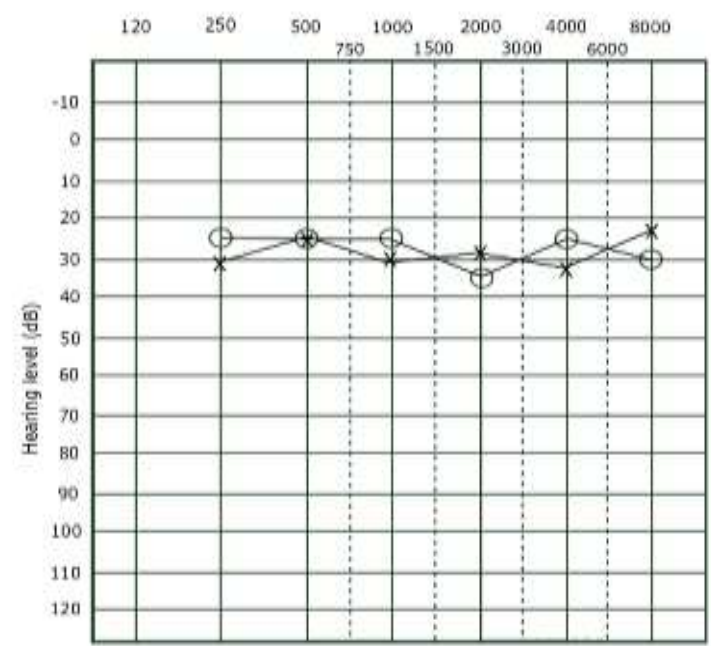

(d)

Figure 4. Audiogram for; (a) Mild hearing loss at high frequencies, (b) Mild to moderate hearing loss at low frequencies, (c) Moderate hearing loss at mid frequencies, and (d) Mild conductive hearing loss [10], [17], [19] 


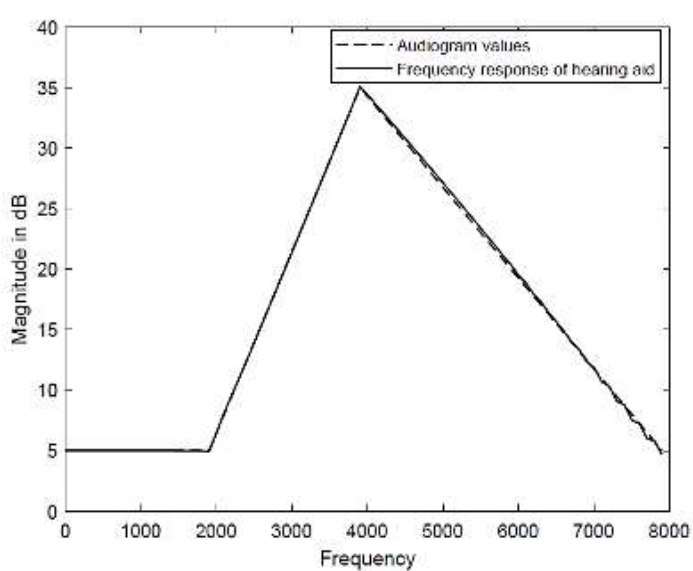

(a)

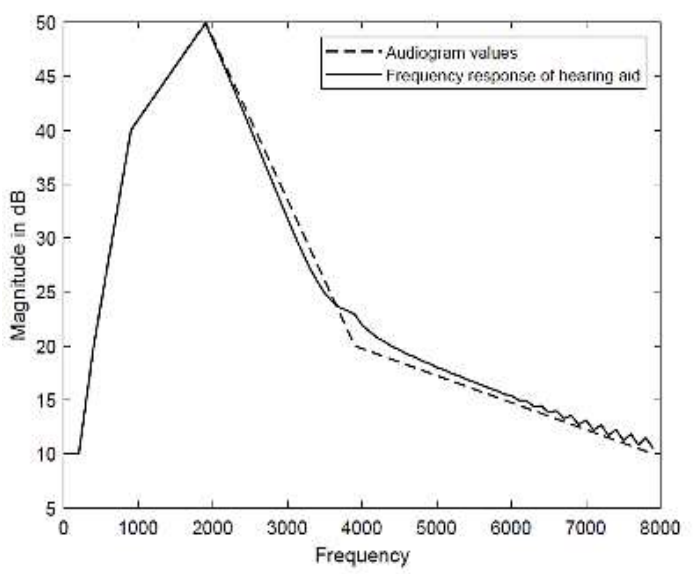

(c)

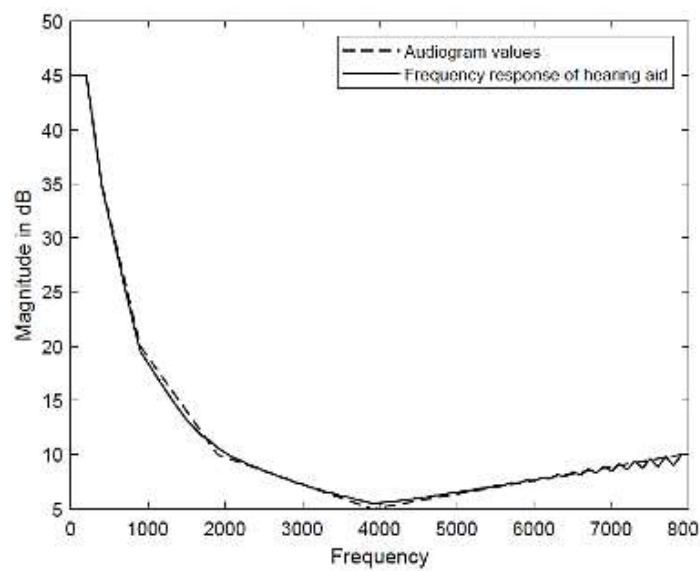

(b)

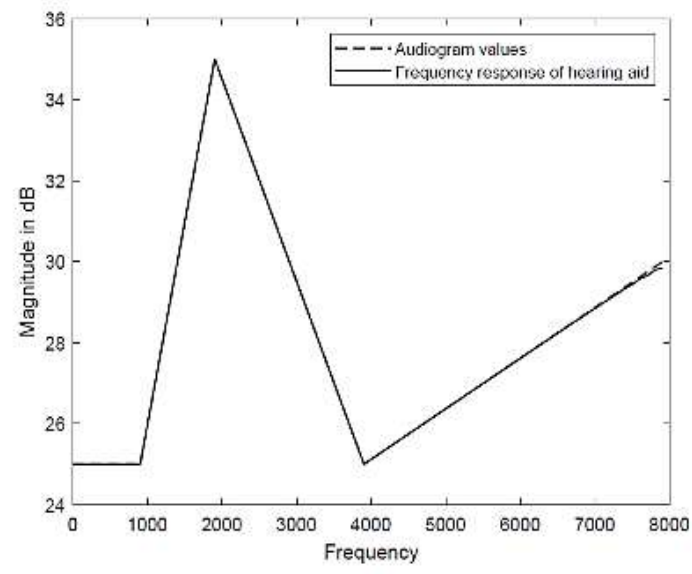

(d)

Figure 5. Audiogram values and the frequency response of hearing aid for; (a) mild hearing loss at highfrequencies, (b) mild to moderate hearing loss at low frequencies, (c) moderate hearing loss at midfrequencies, and (d) mild conductive hearing loss

Table 2. Maximum matching error for different hearing loss cases for given weights and the number ofiterations

\begin{tabular}{|c|c|c|c|c|c|c|c|c|c|c|c|}
\hline \multirow{2}{*}{ Audiogram } & \multirow{2}{*}{ No. of iterat-ions } & \multicolumn{10}{|c|}{ Maximum matching error for given weight } \\
\hline & & 0.1 & 0.2 & 0.3 & 0.4 & 0.5 & 0.6 & 0.7 & 0.8 & 0.9 & 1 \\
\hline \multirow[t]{5}{*}{ Example 1} & 0 & -11.27 & -11.27 & -11.27 & -11.27 & -11.27 & -11.27 & -11.27 & -11.27 & -11.27 & -11.27 \\
\hline & 5 & -11.03 & -10.67 & -10 & -9.2 & -8.34 & -7.4 & -6.62 & -5.82 & -5.1 & -4.44 \\
\hline & 10 & -10.63 & -9.18 & -7.56 & -6.05 & -4.73 & -3.59 & -2.6 & -1.74 & -1.28 & -0.95 \\
\hline & 15 & -9.95 & -7.59 & -5.44 & -3.7 & -2.32 & -1.36 & -0.93 & -0.65 & -0.59 & 0.7 \\
\hline & 20 & -9.18 & -6.14 & -3.76 & -2.01 & -1.07 & -0.67 & 0.49 & 0.7 & 0.76 & 0.72 \\
\hline \multirow[t]{5}{*}{ Example 2} & 0 & -9.03 & -9.03 & -9.03 & -9.03 & -9.03 & -9.03 & -9.03 & -9.03 & -9.03 & -9.03 \\
\hline & 5 & -6.43 & -4.85 & -3.63 & -2.71 & 2.54 & 2.91 & 3.73 & 4.69 & 6.07 & 6.58 \\
\hline & 10 & -4.88 & -2.77 & 1.98 & 2.45 & 2.22 & -1.74 & -3 & -3.67 & 2.13 & 6.17 \\
\hline & 15 & -3.71 & 1.95 & 1.82 & 0.97 & -1.68 & -1.35 & 3.68 & 5.52 & 4.79 & 6.33 \\
\hline & 20 & -2.8 & 1.68 & 0.88 & -1.06 & 1.37 & 1.62 & -3.53 & -3.74 & 6.49 & -3.64 \\
\hline \multirow{5}{*}{ Example 3} & 0 & -14.48 & -14.48 & -14.48 & -14.48 & -14.48 & -14.48 & -14.48 & -14.48 & -14.48 & -14.48 \\
\hline & 5 & -10.9 & -8.88 & -7.3 & -6.14 & -5.08 & -4.22 & -3.45 & 4.6 & 7.11 & 9.55 \\
\hline & 10 & -8.87 & -6.06 & -4.08 & 2.75 & 4.53 & 5.43 & 5.19 & -6.16 & -9.53 & -12.5 \\
\hline & 15 & -7.34 & -4.06 & 2.97 & 3.73 & 2.95 & -5.31 & -6.69 & -6.38 & 16.17 & -11.32 \\
\hline & 20 & -6.03 & -2.52 & 3.14 & 1.87 & -4.53 & 4.12 & 12.53 & 15.9 & 20 & -199 \\
\hline \multirow[t]{5}{*}{ Example 4} & 0 & -2.89 & -2.89 & -2.89 & -2.89 & -2.89 & -2.89 & -2.89 & -2.89 & -2.89 & -2.89 \\
\hline & 5 & 1.89 & 1.9 & 1.86 & 1.77 & 1.64 & 1.48 & 1.3 & 1.1 & 0.92 & 0.75 \\
\hline & 10 & 1.89 & 0.75 & 1.47 & 1.16 & 0.88 & 0.68 & 0.54 & 0.45 & 0.4 & 0.35 \\
\hline & 15 & 1.83 & 1.47 & 1.04 & 0.71 & 0.52 & 0.41 & 0.34 & 0.28 & 0.24 & 0.21 \\
\hline & 20 & 1.73 & 1.18 & 0.73 & 0.49 & 0.37 & 0.29 & 0.24 & 0.2 & 0.17 & 0.15 \\
\hline
\end{tabular}




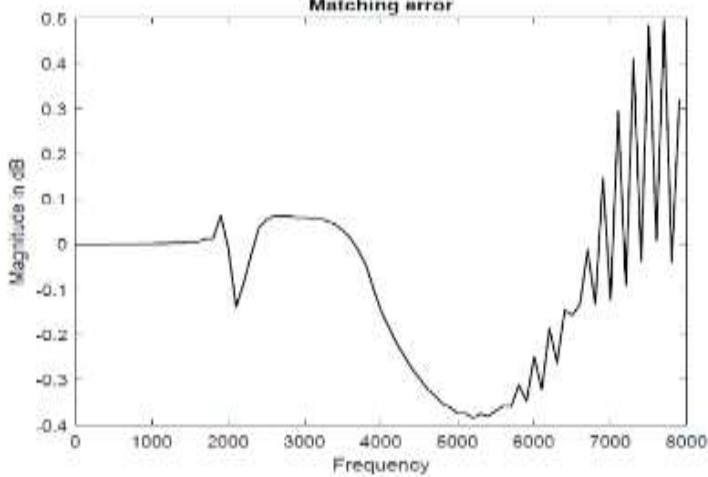

(a)

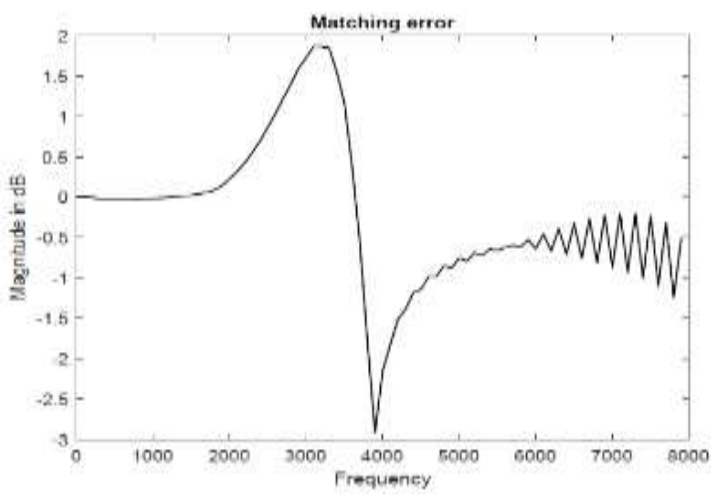

(c)

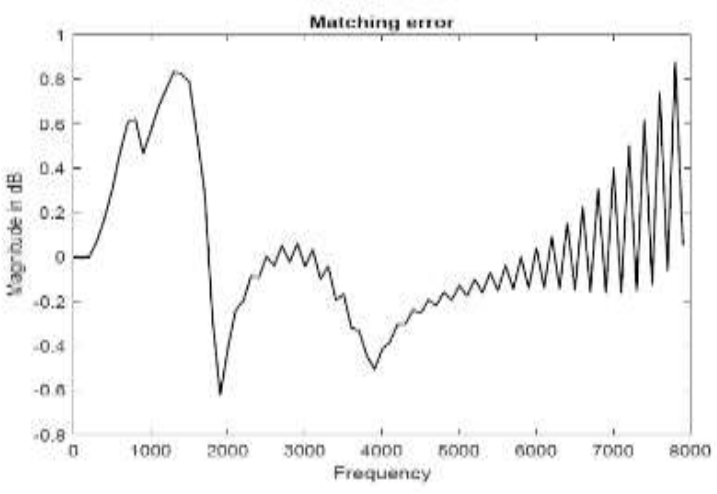

(b)

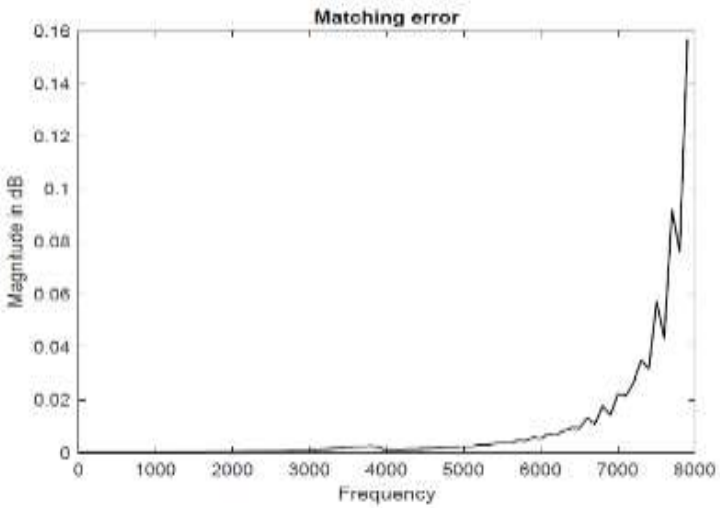

(d)

Figure 6. Matching error for; (a) mild hearing loss at high frequencies, (b) mild to moderate hearing loss atlow frequencies, (c) moderate hearing loss at mid frequencies, and (d) mild conductive hearing loss.

\section{EXPERIMENTAL RESULTS AND ANALYSIS}

Matching errors for different types of audiograms with the different number of iterations and weights are shown in the Table 2. From the Table 2, it is clear that the matching error changes with the number of iterations and weight. In the case of mild hearing loss at high frequency (example 1) better matching can be observed at weight greater than or equals to 0.7 . In the case of mild to moderate hearing loss at low frequency and moderate hearing loss at middle frequency (example 2 and 3) better matching can be observed at weight between 0.3 and 0.7 . Whereas in conductive hearing loss case, better matching can be observed at weight equals to 1 . In all cases matching error reduces with an increase in the number of iterations.

By analyzing Table 2 we can observe that for all cases matching error is better at 20 iterations. If hearing loss is at high frequency weight should be between 0.7 and 0.9 . In case of low frequency hearing loss weight should be between 0.3 and 0.6. From example 3 in case of middle frequency weight should be between 0.4 and 0.7 . For conductive hearing loss weight should be 1 .

The proposed algorithm is tested using an audio signal for the audiogram with mild hearing loss in high frequency as shown in Figure 4. The frequency-domain representation of the input audio signal and the amplified signals are shown in Figure 7 (a). Thus, it is clear that the gain is maximum at the frequencies greater than $2000 \mathrm{~Hz}$ and the gain is changing with the frequency concerning the audiogram. From time-domain waveforms shown in Figure 7 (b), it is clear that signals with high frequency are amplified with high gain values and low-frequency components are amplified with smaller gain values.

\subsection{Delay analysis}

The proposed algorithm comprises three stages DCT, gain adjustment and inverse DCT. Before loading gain values into the hearing aid, Audiogram is re-sampled and modified. The proposed algorithm requires a buffer which is needed to compute the 80-point DCT. Total delay is the delay due to buffer plus delay of three matrix multiplications. According to [24], matrix multiplication with length 80 takes $0.8 \mathrm{~ms}$, three such multiplications are needed for the proposed algorithm, so total delay due to matrix multiplications is $t_{m}=2.4 \mathrm{~ms}$. According to the (4) proposed algorithm takes $5 \mathrm{~ms}$ delay due to the buffer size of 80 at the 
input before applying DCT as shown in Figure 2 (a). From (5) the total delay between input and output signal is $7.4 \mathrm{~ms}$.

$$
T_{b}=\frac{N}{F_{S}}
$$

where $\mathrm{N}$ is the size of the buffer in this case $\mathrm{N}=80$, and $\mathrm{F}_{\mathrm{s}}$ sampling frequency $=16000 \mathrm{~Hz}$. Therefore, $\mathrm{T}_{\mathrm{b}}=5 \mathrm{~ms}$ total delay.

$$
T=T_{b}+T_{m}=5+2.4=7.4 m s
$$

\subsection{Computational efficiency}

To reduce the power consumption and delay hearing aid algorithm should be computationally efficient [25]. In the proposed algorithm DCT and IDCT takes more computations. One dimensional DCT and IDCT requires $2 \mathrm{~N} \log 2(\mathrm{~N})$ number of additions and multiplications, as shown Figure 7 [26]. So, 80-point DCT and IDCT takes 1120 number of multiplications and additions. To adjust the gain values in frequency domain 80 multiplication are needed. To perform the proposed algorithm on 80 samples, 1200 multiplications and 1120 additions are required. Total 14 additions and 15 multiplications are needed for one sample. In [19], the number of multipliers are 67 including all subbands, from this it is clear that multiplier complexity is reduced by $77.61 \%$

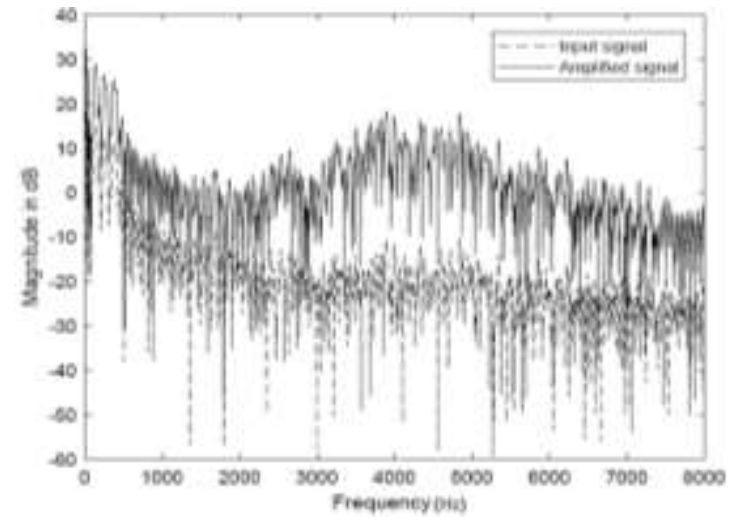

(a)

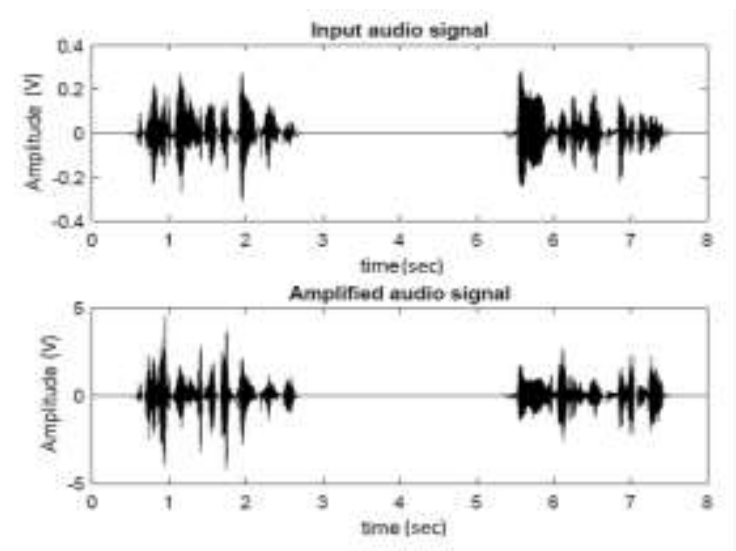

(b)

Figure 7. Input and output of proposed hearing aid algorithm; (a) frequency domain representation and (b) time domain representation

\section{CONCLUSION}

In the present research, a DCT based auditory compensation using uniformly re-sampled and recursively modified audiogram values is implemented. The proposed algorithm provides a simple solution to compensate for the hearing loss without any filter banks and sampling rate conversions. Totally three stages are needed for the whole process: i) Finding DCT for the input audio signal, ii) Gain adjustment and iii) Inverse DCT. DCT coefficients of the audio signal are multiplied with uniformly re-sampled and recursively modified audiogram values to adjust the gains in frequency domain. The performance of the proposed algorithm is compared with different types of filter banks namely uniform filter bank nonuniform filter bank, variable filter bank and reconfigurable filter bank structures. The proposed algorithm is tested for different types of hearing loss cases like mild hearing loss at high frequencies, mild to moderate hearing loss at low frequencies, moderate hearing loss at middle frequencies and mild conductive hearing loss. From the above test, it is illustrated that the proposed DCT based algorithm provides better matching between the frequency response of hearing aid and audiogram. It is achieved with minimum delay and computational complexity.

\section{ACKNOWLEDGEMENTS}

The research leading to these results received funding from Science for Equity, Empowerment and Development Division under Technology Interventions for Disabled and Elderly (TIDE), Department of Science and Technology, Government of India under Grant Agreement No SEED/TIDE/015/2017/G. 


\section{REFERENCES}

[1] N. Tiwari and P. C. Pandey, "Sliding-band dynamic range compression for use in hearing aids," International Journal of Speech Technology, vol. 22, no. 4, pp. 911-926, 2019, doi: 10.1007/s10772-019-09635-4.

[2] W. B. Alshuaib, J. M. Al-Kandari, and S. M. Hasan, "Classification of hearing loss," In Update on Hearing Loss, p. 29, 2015.

[3] Y. D. Hyter and M. B. Salas-Provance, Culturally responsive practices in speech, language, and hearing sciences. Plural Publishing, 2018.

[4] M. C. Killion and S. Fikret-Pasa, "The 3 types of sensorineural hearing loss: Loudness and intelligibility considerations," Hearing journal, vol. 46, pp. 31-31, 1993.

[5] A. R. Møller, Hearing: anatomy, physiology, and disorders of the auditory system. Plural Publishing, 2012.

[6] Y. Lim, "A digital filter bank for digital audio systems," IEEE Transactions on Circuits and Systems, vol. 33, no. 8, pp. 848-849, 1986, doi: 10.1109/TCS.1986.1085988.

[7] T. Lunner and J. Hellgren, "A digital filterbank hearing aid-design, implementation and evaluation," in ICASSP 91: 1991 International Conference on Acoustics, Speech, and Signal Processing.IEEE, 1991, pp. 3661-3664.

[8] E. Onat, M. Ahmadi, G. Jullien, and W. Miller, "Optimized delay characteristics for a hearing instrumentfilter bank," in Proceedings of the 43rd IEEE Midwest Symposium on Circuits and Systems (Cat. No.CH37144), vol. 3, 2000, pp. 1074-1077, doi: 10.1109/MWSCAS.2000.951401.

[9] Y. Wei and Y. Lian, "A computationally efficient non-uniform digital fir filter bank for hearing aid," in IEEE International Workshop on Biomedical Circuits and Systems, 2004, pp. S1-3, doi: 10.1109/BIOCAS.2004.1454116.

[10] Y. Lian and Y.Wei, "A computationally efficient nonuniform fir digital filter bank for hearing aids," IEEE Transactions on Circuits \& Systems I: Regular Papers, vol. 52, no. 12, pp. 2754-2762, 2005, doi: 10.1109/TCSI.2005.857871.

[11] Y.Wei and Y. Lian, "A 16-band nonuniform fir digital filterbank for hearing aid," in 2006 IEEE Biomedical Circuits and Systems Conference, 2006, pp. 186-189.

[12] T.-B. Deng, "Three-channel variable filter-bank for digital hearing aids," IET signal processing, vol. 4, no. 2, pp. 181-196, 2010, doi: 10.1109/ISPACS.2009.4806730.

[13] N. Ito and T.-L. Deng, "Variable-bandwidth filter-bank for low-power hearing aids," in 2010 3rd International congress on image and signal processing, vol. 7, 2010, pp. 3207-3211

[14] R. Mahesh and A. P. Vinod, "Reconfigurable low area complexity filter bank architecture based on frequency response masking for nonuniform channelization in software radio receivers," IEEE Transactionson Aerospace and Electronic Systems, vol. 47, no. 2, pp. 1241-1255, 2011, doi: 10.1109/TAES.2011.5751255.

[15] Y. Wei and D. Liu, "A reconfigurable digital filterbank for hearing-aid systems with a variety of soundwave decomposition plans,” IEEE transactions on Biomedical Engineering, vol. 60, no. 6, pp. 1628-1635, 2013, doi: 10.1109/TBME.2013.2240681.

[16] Amir, A., Rakesh Inani, and Elizabeth Elias., "Reconfigurable low complexity hearing aid system using adjustablefilter bank," in 2016 IEEE Region 10 Conference (TENCON), 2016, pp. 2684-2688, doi: 10.1109/TENCON.2016.7848526.

[17] A. Amir, T. Bindiya, and E. Elias, "Design and implementation of reconfigurable filter bank structure for low complexity hearing aids using 2-level sound wave decomposition," Biomedical Signal Processingand Control, vol. 43, pp. 96-109, 2018, doi: 10.1016/j.bspc.2018.02.020.

[18] Y. Wei, T. Ma, B. K. Ho, and Y. Lian, "The design of low-power 16-band nonuniform filter bank for hearing aids," IEEE transactions on biomedical circuits and systems, vol. 13, no. 1, pp. 112-123, 2018, doi: 10.1109/TBCAS.2018.2888860.

[19] A. Amir, T. Bindiya, and E. Elias, "Low-complexity implementation of efficient reconfigurable structure for costeffective hearing aids using fractional interpolation," Computers \& Electrical Engineering, vol. 74, pp. 391-412, 2019, doi: 10.1016/j.compeleceng.2019.02.008

[20] T. Ma, C. Shen, and Y. Wei, "Adjustable filter bank design for hearing aids system," in 2019 IEEE International Symposium on Circuits and Systems (ISCAS), 2019, pp. 1-5, doi: 10.1109/ISCAS.2019.8702314.

[21] R. S. Rashid and J. R. Mohammed, "Securing speech signals by watermarking binary images in thewavelet domain," Indonesian Journal of Electrical Engineering and Computer Science (IJEECS), vol. 18, no. 2, pp.1096-1103, 2020, doi: 10.11591/ijeecs.v18.i2.pp1096-1103.

[22] M. C. Flynn, R. C. Dowell, and G. M. Clark, "Aided speech recognition abilities of adults with a severeor severeto-profound hearing loss," Journal of speech, language, and hearing research, vol. 41, no. 2,pp. 285-299, 1998, doi: 10.1044/jslhr.4102.285.

[23] Y. Wei and Y. Wang, "Design of low complexity adjustable filter bank for personalized hearing aid solutions," IEEE/ACM Transactions on audio, speech, and language processing, vol. 23, no. 5, pp. 923-931,2015, doi: 10.1109/TASLP.2015.2409774.

[24] P. Saha, A. Banerjee, P. Bhattacharyya, and A. Dandapat, "Improved matrix multiplier design for high speed digital signal processing applications," IET circuits, devices \& systems, vol. 8, no. 1, pp. 27-37, 2014.

[25] S. Ashraf, M. Gao, Z. Chen, H. Naeem, A. Ahmad, and T. Ahmed, "Underwater pragmatic routing approach through packet reverberation mechanism," IEEE Access, vol. 8, pp. 163 091-163 114, 2020, doi: 10.1109/ACCESS.2020.3022565.

[26] M. St'ephane, "Chapter 8-wavelet packet and local cosine bases," A Wavelet Tour of Signal Processing(Third Edition), pp. 377-434, 2009. 